\title{
Improving Blood Flow Simulations by Incorporating Measured Subject-Specific Wall Motion
}

Jonas Lantz, Petter Dyverfeldt and Tino Ebbers

\author{
Linköping University Post Print
}

\section{Tweet}

N.B.: When citing this work, cite the original article.

Original Publication:

Jonas Lantz, Petter Dyverfeldt and Tino Ebbers, Improving Blood Flow Simulations by Incorporating Measured Subject-Specific Wall Motion, 2014, Cardiovascular engineering and technology, (5), 3, 261-269.

http://dx.doi.org/10.1007/s13239-014-0187-5

Copyright: (C) Springer International Publishing AG, Part of Springer Science+Business Media http://link.springer.com/

Postprint available at: Linköping University Electronic Press

http://urn.kb.se/resolve?urn=urn:nbn:se:liu:diva-107479 


\section{Title page}

Title: Improving Blood Flow Simulations by Incorporating Measured Subject-Specific Wall Motion (87 characters)

Abbreviated title: incorporating wall motion in blood flow simulations (51 characters)

Authors: Jonas Lantz (a,d), Petter Dyverfeldt (b,c) Tino Ebbers (a,b,c,d)

a) Department of Science and Technology, Linköping University

b) Department of Medical and Health Sciences, Linköping University

c) Center for Medical Image Science and Visualization (CMIV), Linköping University

d) Swedish e-Science Research Centre (SeRC)

Address for correspondence:

Jonas Lantz, IEI, Linköping University, SE-581 83 Linköping, Sweden

Phone: +4613282335, email: Jonas.Lantz@liu.se, fax: +4613281101 


\section{Abstract}

Purpose: Physiologically relevant simulations of blood flow require models that allow for wall deformation. Normally a fluid-structure interaction (FSI) approach is used; however, this method relies on several assumptions and patient-specific material parameters that are difficult or impossible to measure in vivo.

Method: In order to circumvent the assumptions inherent in FSI models, aortic wall motion was measured with MRI and prescribed directly in a numerical solver. In this way is not only the displacement of the vessel accounted for, but also the interaction with the beating heart and surrounding organs. In order to highlight the effect of wall motion, comparisons with standard rigid wall models was performed in a healthy human aorta.

Results: The additional computational cost associated with prescribing the wall motion was low (17\%). Standard hemodynamic parameters such as time-averaged wall shear stress and oscillatory shear index seemed largely unaffected by the wall motion, as a consequence of the smoothing effect inherent in time-averaging. Conversely, instantaneous wall shear stress was greatly affected by the wall motion; the wall dynamics seemed to produce a lower wall shear stress magnitude compared to a rigid wall model. In addition, it was found that if wall motion was taken into account the computed flow field agreed better with in vivo measurements.

Conclusion: This article show that it is feasible to include measured subject-specific wall motion into numerical simulations, and that the wall motion greatly affects the flow field. This approach to incorporate measured motion should be considered in future studies of arterial blood flow simulations.

\section{Key terms}

Computational fluid dynamics, Magnetic resonance imaging, Fluid-structure interaction, aorta, time averaged wall shear stress, prescribed wall motion, 


\section{Introduction}

There is a close relationship between blood flow and many terms of cardiovascular diseases [1]. In order to understand the genesis and progression of these diseases, accurate description and assessment of blood flow features are crucial. With numerical modeling it is possible to compute hemodynamic parameters that are otherwise not possible to obtain in vivo. For example, it appears that the patchy distribution of atherosclerotic lesions is caused by local features in the blood flow; disturbed or even turbulent flows seem to have different effects compared to steady laminar flows [2-4]). In order to quantify the effect of the flow on arterial endothelial cells, a number of hemodynamic indices has been put forward, the most common reported in the literature being the time-averaged wall shear stress (TAWSS) and the Oscillatory Shear Index (OSI). In vivo measurements of arterial WSS using current clinical imaging modalities suffer from accuracy issues [5-6], therefore modeling is the main approach to WSS estimation. In addition, individual variability makes it difficult to use imaging and experiences from larger groups to provide information on a single individual patient [7]. Arterial geometry is probably the single most important determinant of local blood flow patterns, and modeling the interaction between the blood flow and the arterial wall represents one of the major challenges in the field of computational hemodynamics [8]. As noted by Barakat [9], there is a critical need for systematic investigations on how vessel geometry in numerical models affects arterial flow fields. The simulations used for WSS assessment typically assume that the arterial walls are rigid, which greatly simplifies the simulation process. The effect of wall motion on velocity and WSS fields is often assumed to be negligible. This may be reasonable in older subjects and certain patient groups with increased vascular stiffness $[1,10]$. However, vessel walls are typically not rigid and such an assumption will introduce a mismatch between inlet and outlet flow rates, making a direct comparison with in vivo measurements difficult. The limitation introduced by this mismatch in flow can only be overcome with a model that accounts for wall distensibility.

Fluid-structure interaction (FSI) models compute the aortic wall deformation due to changes in aortic pressure, and can yield physiological pulse wave forms and pressures [8]. However, these models are based on several assumptions including the wall thickness, the stress-strain relationship of the arterial wall, homogeneous material properties, the impact of surrounding tissue and organs, and pressure and mass wave reflections from arterial bifurcations and branches outside the computational domain. Depending on desired model complexity and available data on the subject/patient, the wall can be modeled using either a linear [11] or non-linear $[12,13]$ material model. 
However, to obtain an accurate wall deformation for non-linear material models, the residual stresses, that are present even in a non-pressurized aortic wall, must be accounted for. Furthermore, the descending aorta is longitudinally tethered by the spine, while the ascending aorta is less constrained by external tissues and structures [14], which will also affect the wall motion. In addition, the translation of the wall resulting from the motion of the beating heart is often neglected, even though it can have a significant impact on the flow field [15].

The uncertainties included in FSI models pose a great obstacle when performing patient-specific simulations. However, as noted by Taylor and Figueroa [16], new imaging techniques permit the direct characterization the motion of the vessel wall. Consequently, the limitations of current FSI models may be overcome by directly prescribing measured wall motion in the numerical model. Magnetic resonance imaging (MRI) has earlier been used to measure arterial wall motion: Jin et al. [15] prescribed the wall motion from two through-plane MRI measurements in the ascending aorta, and performed interpolation in between the two planes while keeping the aortic arch and descending aorta rigid. Their conclusion was that the computed flow field only agreed with the measured flow field when the full wall motion (displacement due to pressure changes and translation due to the beating heart) was included, suggesting that the flow field is not only dependent on the geometry but also on the motion of the aorta. In a study by Torii et al [17], the motion of the right coronary artery over 14 time points in the cardiac cycle was measured and implemented in a numerical solver. The MRI data acquisition in their study required seven separate scanning sessions, each lasting 75-90 minutes, over a period of a month. Their conclusions were that the wall motion affected the instantaneous wall shear stress and that vessel motion measured by MRI could be incorporated in numerical solvers.

The goal of this study was to measure the full three-dimensional wall motion of a healthy human aorta over the entire cardiac cycle using a single MRI measurement, and incorporate this information into a numerical model. This allows us to investigate the impact of wall motion on hemodynamics, as well as the importance of including wall motion in image-based CFD models. Incorporating the three-dimensional wall motion in the model does not only address subject-specific wall displacement, but does also include the effects of translational vessel motion caused by interaction with surrounding organs and tissues. To demonstrate the effect of the wall dynamics, a comparison with rigid wall models was performed. 


\section{Method}

\subsection{MRI Acquisition}

Time resolved-wall motion and flow were acquired with a three-dimensional cine balanced steady-state free precession (bSSFP) sequence on a 1.5 T Philips Achieva MRI scanner (Philips Healthcare, Best, the Netherlands). A sagittal slab orientation in the "candy-cane view" was prescribed to cover the thoracic aorta. Imaging parameters included flip angle $=80$ degrees, pixel bandwidth $=1325 \mathrm{~Hz}, \mathrm{TR} / \mathrm{TE}=4.0 / 2.0 \mathrm{~ms}$, and $\mathrm{k}$-space segmentation factor

$=11$ (temporal resolution $=44 \mathrm{~ms})$. The scanned subject had a heart rate of $56 \mathrm{bpm}$ and 25 frames evenly distributed throughout the complete cardiac cycle were reconstructed. Additional imaging parameters included a matrix size of 176 x 176, 30 slices and a three-dimensional field-of-view of $300 \times 300 \times 60 \mathrm{~mm}$ (voxel size $=1.7 \times 1.7$ × $2 \mathrm{~mm}$ ). The scan time was 480 heartbeats, or about 8 minutes and 30 seconds. In addition, through-plane velocity profiles were measured in the ascending and descending aorta, as well as in a plane through the three branching vessels of the aortic arch. Imaging parameters were as follows. Ascending and descending aorta: Pixel size $=1.56 \times 1.56 \mathrm{~mm}$, slice thickness $=7 \mathrm{~mm}$, temporal resolution $=30 \mathrm{~ms}, \mathrm{VENC}=200 \mathrm{~cm} / \mathrm{s}, \mathrm{TR} / \mathrm{TE}=4.98 / 2.96 \mathrm{~ms}$, pixel bandwidth $=498$ $\mathrm{Hz}, \mathrm{k}$-space segmentation factor $=3$. Neck: Pixel size $=1.56 \times 1.56 \mathrm{~mm}$, slice thickness $=6 \mathrm{~mm}$, temporal resolution $=27 \mathrm{~ms}, \mathrm{VENC}=150 \mathrm{~cm} / \mathrm{s}, \mathrm{TR} / \mathrm{TE}=6.63 / 3.94 \mathrm{~ms}$, pixel bandwidth $=342 \mathrm{~Hz}$, k-space segmentation factor $=2$.

\subsection{Numerical Model}

The three-dimensional aortic wall was delineated in all 25 time frames of the MR data, yielding time-resolved aortic geometry at $44 \mathrm{~ms}$ temporal resolution. The vessel wall delineation was performed using a freely available software (Segment Heiberg et al [18]), and was improved using manual corrections at a few locations. A high quality mesh was constructed in ANSYS ICEM 14.5 for the first geometry, with 3 million anisotropic hexahedral cells. A fine near-wall resolution was obtained by gradually letting the first layer thickness grow with a expansion factor of 1.1 until the cell size matched the bulk mesh. A sensitivity study on meshes with 3 and 5 million cells was performed, and the difference in velocity and wall shear stress was less than $4 \%$, at it was determined that the mesh with 3 million cells was sufficient for this study. 
In order to minimize the additional computational cost of including wall motion, the topology of the initial mesh was kept constant and stretched to match the subsequent geometries. In this way re-meshing was not needed, and the cost of interpolating flow variables between different topologies could be avoided. The mesh quality was monitored to ensure that no negative or low-quality cells were obtained.

The pulsatile flow inside the aorta was simulated using ANSYS CFX 14.5. The fluid was assumed to be Newtonian with a viscosity of $3.5 \mathrm{e}-3 \mathrm{~Pa}$.s and incompressible with a density of $1060 \mathrm{~kg} / \mathrm{m} 3$. The time step in the simulation was $0.0044 \mathrm{~s}(1 / 10$ of the time resolution of the MRI wall motion measurement), and piecewise cubic hermite spline interpolation was performed in time to obtain intermediate geometries for each time step in the flow simulation. The mesh motion was incorporated through a user defined FORTRAN subroutine in CFX, and called at the beginning of each time step. For details on the mesh interpolation, see the supplementary materials. The numerical schemes for the advection and temporal terms were second order accurate and convergence was obtained when the residuals had decreased 5 orders of magnitude and global imbalances of mass and momentum were less than $0.1 \%$. Typically 5 to 6 iterations per time step were required. Four cardiac cycles were simulated, but results were only taken from the last cycle to ensure that initial transient effects had disappeared.

The measured in-plane velocity profile in the ascending aorta and branching vessels were used as boundary conditions in the model, see Figure 1. As the temporal resolution of the simulation was higher than the measurement, piecewise cubic hermite spline interpolation was performed in the temporal domain to obtain values for the boundary condition for all time steps. In the descending aorta a constant static pressure boundary condition was set. Besides the prescribed motion, the aortic wall was considered non-deformable and to obey the no-slip condition. In order to investigate the influence of wall motion, five different cases were simulated: three cases with wall motion and two rigid wall cases. A simulation with all 25 measured geometries was considered as the baseline case since it used all information available. A second case used every third measured geometry, resulting in nine geometries in total, while a third case used only two measurements: the first geometry (minimum volume) and the 6th geometry (maximum volume), see Figure 2. Finally, two rigid wall simulations were performed: one simulation with the smallest aortic volume and one simulation with the largest volume. In this way the influence of the size of the vessel could be deducted, as well as how the dynamics of the wall motion affected the flow field.

[Figure 1 about here] 
[Figure 2 about here]

\subsection{Post-processing}

The CFD-results were post-processed in Matlab, and time-averaged wall shear stress (TAWSS) and oscillatory shear index (OSI) were computed. The TAWSS variable is simply the average WSS magnitude ( $\square_{\mathrm{w}}$ ) over one cardiac cycle, defined as:

$$
\mathrm{TAWSS}=\frac{1}{T} \int_{0}^{T} \tau_{w} d t(1)
$$

while the OSI variable is defined as:

$$
\mathrm{OSI}=0.5\left(1-\frac{\left|\frac{1}{T} \int_{0}^{T} \tau_{w} d t\right|}{\frac{1}{T} \int_{0}^{T}\left|\tau_{w}\right| d t}\right)
$$

where $\mathrm{T}$ is the time for one cardiac cycle. OSI describes the cyclic departure of the WSS vector from its predominant axial alignment $[19,20]$, and values can range from zero to 0.5 , where zero means that the instantaneous WSS vector is aligned with the time-averaged WSS vector throughout the cardiac cycle. A value of 0.5 implies that the instantaneous WSS vector never is aligned with the time-averaged vector, indicating a very oscillatory behavior. OSI is insensitive to shear magnitude and must therefore be used with caution; a large OSI value can indicate a disturbed flow region with high or low WSS magnitudes. In addition to WSS variables, velocity profiles in the descending aorta were compared with MRI measurements.

\section{Results}

The translational motion of the ascending aorta can be quite complex, as shown in Figure 3. It can be seen that the aorta first moves toward the right side of the body and then anteriorly during systole, and then returning slowly back in a left-posterior direction to the original position during diastole. At $0.75 \mathrm{~s}$ there is a sudden movement, which could be an effect of atrial contraction. The total distance traveled by the centroid is over $20 \mathrm{~mm}$, which is on the order of an aortic diameter. The effect of only including two geometries is apparent on the translation; the translation appears straight and does not account for the complex motion in the simulation with 25 measurements.

[Figure 3 about here] 
Even though only a single aorta has been studied, the modeling is subject-specific and the velocity profiles in the descending aorta can be validated by direct comparisons with measured in vivo data. Qualitatively, the simulations with prescribed wall motion results in velocity profiles that better match the MRI measurements, compared to the rigid wall simulations, see Figures 4 and 5. It can also be seen that the two rigid wall simulations give different results, as a result of the difference in volume. The case with rigid wall and maximum volume (Rigid Max Vol.) has a lower mean velocity compared to the case with rigid wall and minimum volume (Rigid Min Vol.). This is due to the larger volume and that conservation of mass effectively decreases the mean velocity in the cross-section. Similarly, the Rigid Min Vol. case over predicts the velocity magnitude compared to MRI measurements. In addition, the shape of the velocity profile is different in the rigid wall cases compared to the prescribed wall and MRI results; in general there is a better agreement between MRI measurements and numerical simulations with prescribed wall motion.

[Figures 4 and 5 about here]

[Figure 6 about here]

This is also highlighted in Figure 6, where the flow rate through a plane in the descending aorta for each case is plotted. Table 1 summarizes the peak flow rate and when it occurs for each case. The wall motion seems to have less effect on the TAWSS and OSI parameters, indicated by the close resemblance between the aortas in Figure 7. Considering the TAWSS, the rigid-wall simulation with the smallest volume experienced elevated values compared to the other simulations, which could be an effect of the smaller volume creating higher velocities, and thus, also higher shear rates closer to the wall. On the other hand, for the OSI parameter the rigid simulation with the largest volume experienced elevated values compared to the other simulations. This could be an effect of an overall lower velocity magnitude which could increase wall shear stress oscillation.

[Figure 7a, 7b about here]

To assess whether wall motion affected instantaneous WSS values, the WSS magnitude at peak flow rate for all cases was studied, see Figure 8 . The cases with wall motion exhibits a different WSS pattern and lower magnitude compared to the rigid wall cases. In addition, locations with high WSS values in the cases with prescribed wall motion experiences even higher values when the wall is considered rigid. In particular the case with the smallest domain volume has the highest WSS values, again an effect of the smaller volume creating higher velocities and shear rates. 
[Figure 8 about here]

The additional computational resources required for reading, interpolating and writing new meshes were considered, and it was found that incorporating all 25 measured geometries in the simulation increased the time for simulating one cardiac cycle by $17 \%$ compared to a standard rigid wall simulation, see Table 2 . For nine geometries the additional time was $13 \%$, while for only two geometries it was $7 \%$.

\section{Discussion}

This study introduced blood flow simulations with prescribed subject-specific in vivo wall motion measured by 4DMRI. Traditional simulations of aortic flows normally assume rigid walls or compute the wall displacement through a fluid-structure interaction simulation. The assumption of rigid walls neglects the dynamics that is introduced by the moving wall, while FSI simulations require knowledge of material parameters and in vivo boundary conditions that are often unknown. The translation of the aorta is rarely accounted for, and as the number of uncertainties increase, the accuracy of the simulation result may become degraded. A FSI simulation of the model was not performed, as subject-specific wall material properties were not available and using standard values from literature would only add uncertainty to the results. A range of different wall parameters and different values of the wall thickness would therefore have to be simulated and compared to the measured wall motion, but this was not the scope of the study. Instead, the method and results presented in this study shows that these hurdles can be overcome by measuring the wall motion using MRI and directly prescribe it in the numerical simulation. However, it should be noted that the MRI measurements of time-resolved 3D vascular geometry provides information that enables inverse modeling for determination of wall properties. In this way local wall stiffness can be computed, which would increase the accuracy of an FSI simulation.

The additional computational cost involved in prescribing the wall motion comes from reading new meshes and interpolating coordinates for each time step in the numerical simulation. However, with a single cardiac cycle only taking 3 hours for a rigid wall simulation, the additional cost of involving a prescribed wall motion was only 30 minutes. This can certainly be considered affordable, as the physiological relevance increases considerably. In fact, MRI acquisition, segmentation, meshing, and setup of the CFD-model takes longer time, making the additional time required for including wall motion negligible. Note that the extra effort of meshing 25 geometries did not take 25 
times longer compared to when meshing only a single geometry; much of the initial work could be scripted and reused. The mesh motion subroutine used in the simulation software can probably be optimized even further, decreasing the additional computational effort even more.

The translation of the ascending aorta is complex, as a result of the motion from the beating heart. The measured motion agreed very well with the results from the study of Jin et al. [15], both in shape and distance. They also measured a sudden change in the translation at the end of diastole, which probably originates from atrial contraction as it occurs at the P-wave in the electrocardiogram. Flow forces may also introduce a translating motion, but this effect is most likely small compared to the effect from the heart motion. Conservation of mass together with a rigid wall assumption implies that the amount of mass that enters the domain in the ascending aorta must at the same time leave it in the branching vessels in the aortic arch and in the abdominal aorta. This is not the case in vivo where the aortic volume changes. Instead, the aorta acts as an elastic reservoir, when the aortic wall distends due to increased aortic pressure. This Windkessel effect will affect the local flow field as mass is stored and removed at different locations and times, compared to when the aortic walls are considered rigid. This effect can be seen in the velocity profiles found in Figures 4 and 5, and is more pronounced in Figure 6 where the flow rates for each case are plotted. The effect of the wall motion is obvious, as the rigid wall assumption results in a totally different flow rate curve compared to the measurement. The lack of distensibility in a rigid wall model forces more blood through the model during systole, while the models that account for wall motion will store parts of the blood volume and release it later in the cardiac cycle. It is also seen in Figure 6 that the models that use all 25 measurements and 9 measurements agrees better with MRI data, compared to the case which only used 2 measurements. Incorporating only 2 geometries was apparently insufficient to accurately capture the Windkessel -effect. This is because the local wall displacement was not captured accurately enough in the interpolation, compared to when including more geometries. Both the shape and magnitude of the velocity profile in the descending aorta changed when incorporating wall motion and the agreement with in vivo measurements improved considerably. Clearly, the interaction between the wall and the flow will affect the hemodynamics. There are small differences in the shape of the velocity profile between the baseline case and the two cases with fewer geometries. This indicates that the method works for 4D morphological data with relatively low temporal resolution, which in turn would shorten scan time. The small differences between the MRI 
velocity profiles and the baseline CFD case could be to uncertainties in boundary condition specification, MRI measurement and segmentation of the wall.

The time-averaged WSS and oscillatory shear index are both time-integrated quantities - as such, time dependent effects may be filtered out and diminished in the results. This can be seen in the results for TAWSS and OSI, where the prescribed wall motion simulations yield very similar results. This is in line with a previous study [11] where it was concluded that the time-averaging the WSS parameters effectively filters out any temporal effects. On the other hand, the instantaneous WSS magnitude is affected by the wall motion: at peak flow rate both rigid wall cases result in higher WSS values compared to the three cases with prescribed wall motion. Instantaneous WSS, as well as timeaveraged WSS and oscillatory shear index have been suggested to play a role in the development of atherosclerosis [1-4]. If rigid-wall CFD is used to find thresholds for when atherosclerosis can develop, then those thresholds values might be inaccurate. This study shows that a rigid wall assumption might be questionable if one is interested in instantaneous WSS values.

\section{Conclusions}

Incorporating subject-specific wall motion in blood flow simulations results in considerable improvement of the computed flow field compared to in-vivo measurements. The proposed approach is straight forward to implement, and the additional computational costs compared to usage of a rigid wall model is relatively small. With the inclusion of measured subject-specific wall motion, the physiological relevance of simulations of arterial blood flow increases, and can lead to novel insights in vascular blood flow dynamics.

\section{Acknowledgements}

This study was funded by the Swedish e-Science Research Centre, the Centre for Industrial Information Technology, the Swedish Research Council, and the European Research Council. The Swedish National Infrastructure for Computing is acknowledged for computational resources provided by the National Supercomputer Centre.

\section{Conflict of interest}


The authors declared that they have no conflict of interest.

\section{Statement of Human studies}

All procedures followed were in accordance with the ethical standards of the responsible committee on human experimentation (institutional and national) and with the Helsinki Declaration of 1975, as revised in 2000 (5). Informed consent was obtained from the subject for being included in the study.

\section{Statement of Animal Studies}

No animal studies were carried out by the authors for this article 


\section{References}

[1] Steinman, D.A. Image-based computational fluid dynamics modeling in realistic arterial geometries. Annals of biomedical engineering 30, 483-497, 2002

[2] Malek, A.M., Alper S.L, and Izumo S, Hemodynamic shear stress and its role in atherosclerosis. JAMA: the journal of the American Medical Association 282, 2035-2042, 1999

[3] Resnick, N., Yahav H, Shay-Salit A Shushy M, Schubert S, Zilberman L.C.M, and Wofovitz E., Fluid shear stress and the vascular endothelium: for better and for worse. Progress in biophysics and molecular biology 81, 177199,2003

[4] Assemat, P. and Hourigan K, Evolution and rupture of vulnerable plaques: a review of mechanical effects. ChronoPhysiology and Therapy 3, 23-40, 2013

[5] Mynard, J.P., Wasserman B.A, and Steinman D.A., Errors in the estimation of wall shear stress by maximum Doppler velocity. Atherosclerosis 227, $259-266$.

[6] Petersson, S., Dyverfeldt P, and Ebbers T.. Assessment of the accuracy of MRI wall shear stress estimation using numerical simulations. Journal of Magnetic Resonance Imaging 36, 128-138, 2012

[7] Taylor, C.A. Draney M.T, Ku J.P, Parker D, Steele B.N, Wang K and Zarins C.K. Predictive medicine: computational techniques in therapeutic decision-making. Computer Aided Surgery 4, 231-247, 1999.

[8] Caballero, A. and. Laín S., A review on computational fluid dynamics modelling in human thoracic aorta. Cardiovascular Engineering and Technology , 1-28, 2013

[9] Barakat, A.I., Blood flow and arterial endothelial dysfunction: Mechanisms and implications. Comptes Rendus Physique 14, 479 - 496, 2013 
[10] Taylor, C.A., and Steinman D.A. Image-based modeling of blood flow and vessel wall dynamics: applications, methods and future directions. Annals of biomedical engineering 38, 1188-1203, 2010.

[11] Lantz, J., Renner J, and Karlsson M, Wall shear stress in a subject specific human aorta - influence of fluidstructure interaction. International Journal of Applied Mechanics 3, 759-778, 2011

[12] Gao, F., Guo, Z., Sakamoto, M and T. Matsuzawa, Fluid-structure interaction within a layered aortic arch model. Journal of biological physics 32, 435-454., 2006

[13] Khanafer, K., Bull J, and R. Berguer. Fluid-structure interaction of turbulent pulsatile flow within a flexible wall axisymmetric aortic aneurysm model. European Journal of Mechanics-B/Fluids 28, 88-102., 2009

[14] Moireau, P., Xiao N, Astorino M, Figueroa CA, Chapelle D, Taylor C.A., and Gerbeau J.F.. External tissue support and fluid-structure simulation in blood flows. Biomechanics and modeling in mechanobiology 11, 1-18, 2012

[15] Jin, S., Oshinski J, and Giddens D.P, Effects of wall motion and compliance on flow patterns in the ascending aorta. Journal of biomechanical engineering 125, 347-354., 2003

[16] Taylor, C.A and Figueroa C. Patient-specific modeling of cardiovascular mechanics. Annual review of biomedical engineering 11, 109-134, 2009.

[17] Torii, R. Keegan J , Wood N.B, Dowsey A.W, Hughes A. D, Yang G-Z, Firmin DN, Thom S.A and Xu X.Y, MR image-based geometric and hemodynamic investigation of the right coronary artery with dynamic vessel motion, Annals of biomedical engineering, 38, 2606-2620, 2010 
[18] Heiberg, E., Sjogren J., Ugander M, Carlsson M, Engblom H, and H Arheden, Design and validation of Segment-freely available software for cardiovascular image analysis. BMC Med Imaging 10, 1, 2010

[19] Ku, D.N., Giddens DP, Zarins C.K, and Glagov S. Pulsatile flow and atherosclerosis in the human carotid bifurcation. Positive correlation between plaque location and low oscillating shear stress. Arteriosclerosis 5, $293-$ 302. 1985

[20] He, X., and Ku D.N, Pulsatile flow in the human left coronary artery bifurcation: average conditions. J

Biomech Eng 118, 74-82., 1996 


\section{Tables}

Table 1: Peak flow rate and when peak flow rate occurs for each case.

\begin{tabular}{|l|l|l|}
\hline Case & Peak Flow Rate $[\mathrm{ml} / \mathrm{s}]$ & Time [s] \\
\hline MRI Measurement & 454 & 0.16 \\
\hline Rigid wall & 567 & 0.07 \\
\hline 2 Measurements & 433 & 0.07 \\
\hline 9 Measurements & 436 & 0.14 \\
\hline 25 Measurements & 430 & 0.20 \\
\hline
\end{tabular}


Table 2: Wall clock time for one cardiac cycle for each case using 40 CPU cores

\begin{tabular}{|l|l|l|l|}
\hline Simulation case & \# of meshes & Time $[\mathrm{h}]$ & Additional time \\
\hline Rigid wall min volume & 1 & 3.0 & - \\
\hline Rigid wall max volume & 1 & 3.0 & - \\
\hline Prescribed wall motion & 2 & 3.2 & $7 \%$ \\
\hline Prescribed wall motion & 9 & 3.4 & $13 \%$ \\
\hline Prescribed wall motion & 25 & 3.5 & $17 \%$ \\
\hline
\end{tabular}


Figures

Figure1_Lantz.eps

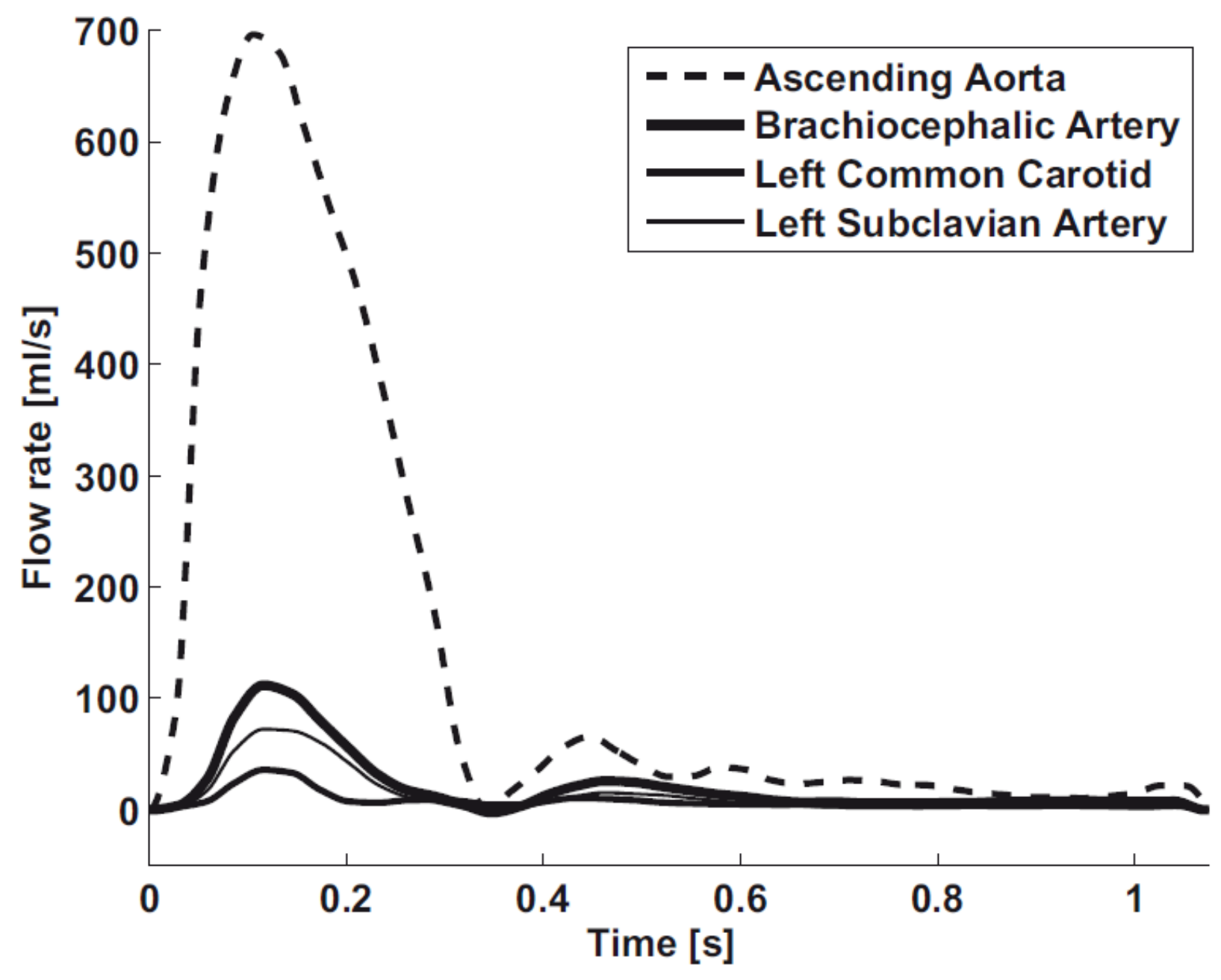


Figure2_Lantz.eps

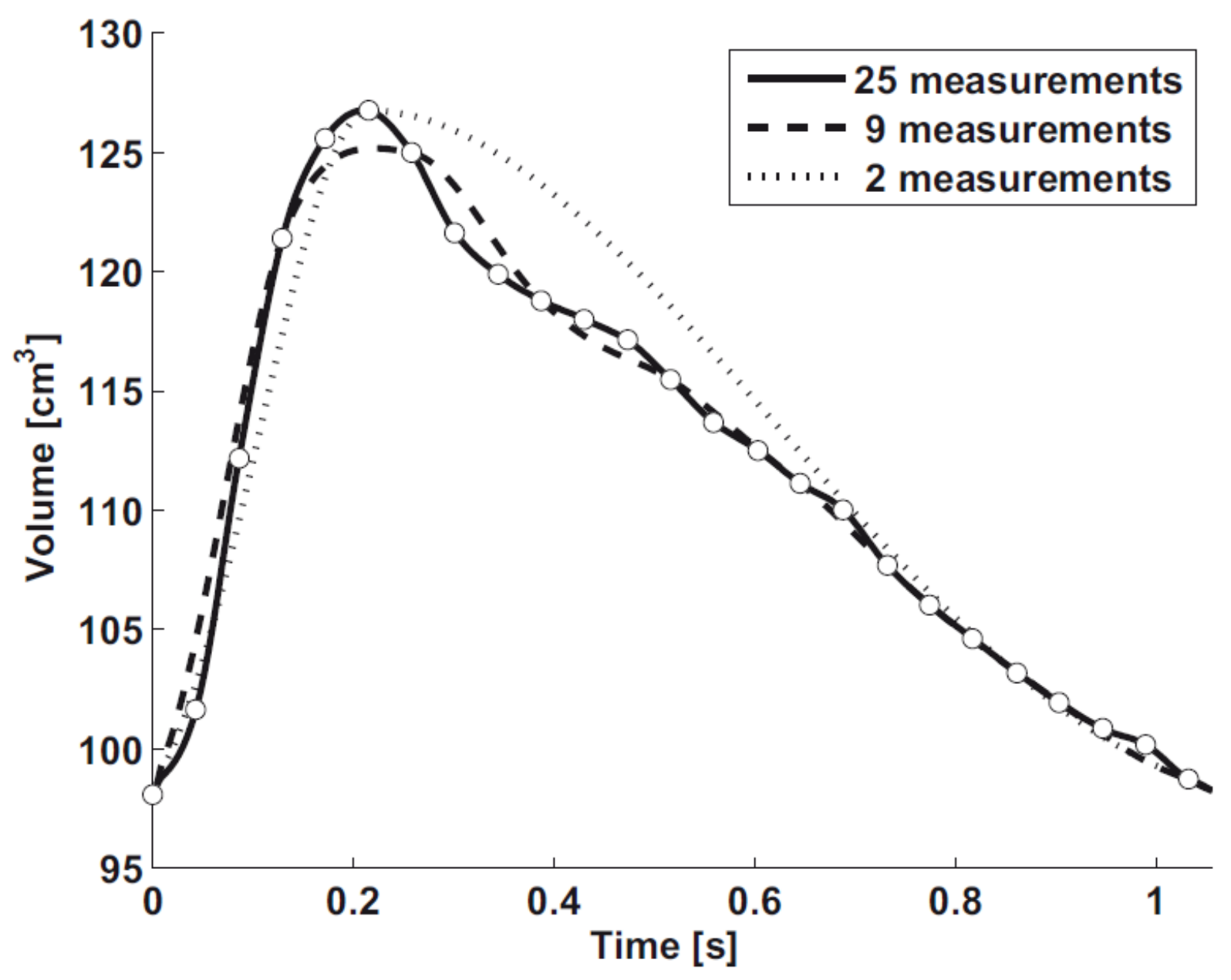


Figure3_Lantz.eps

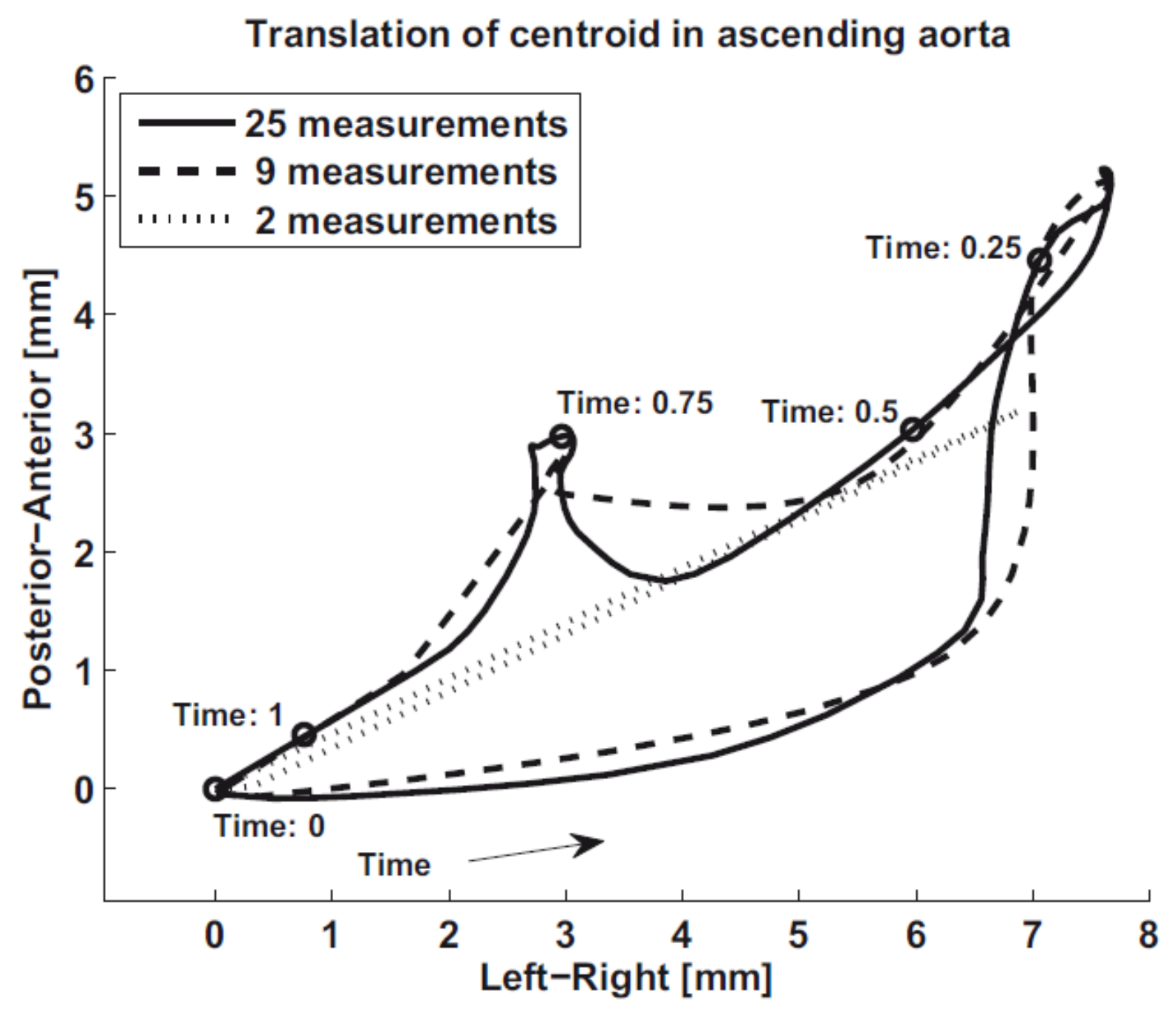


Figure4_Lantz.eps

Figure5_Lantz.eps
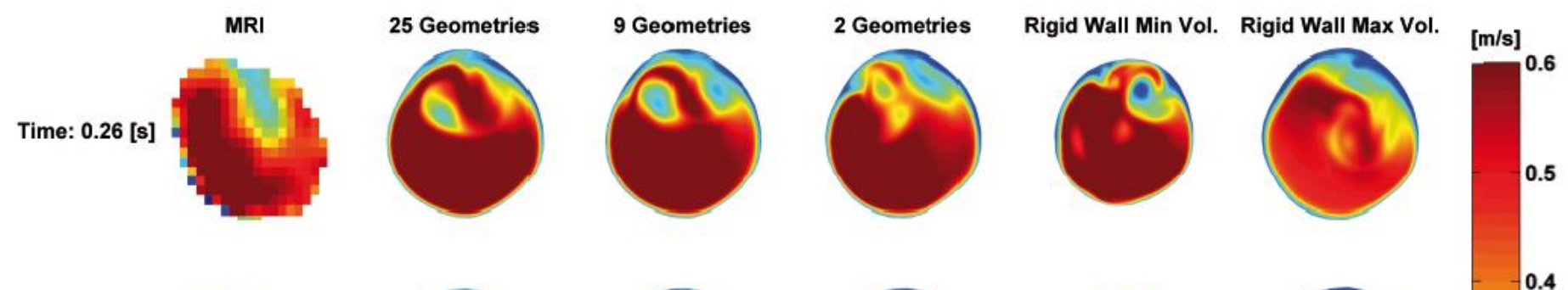

Time: $\mathbf{0 . 2 8}$ [s]
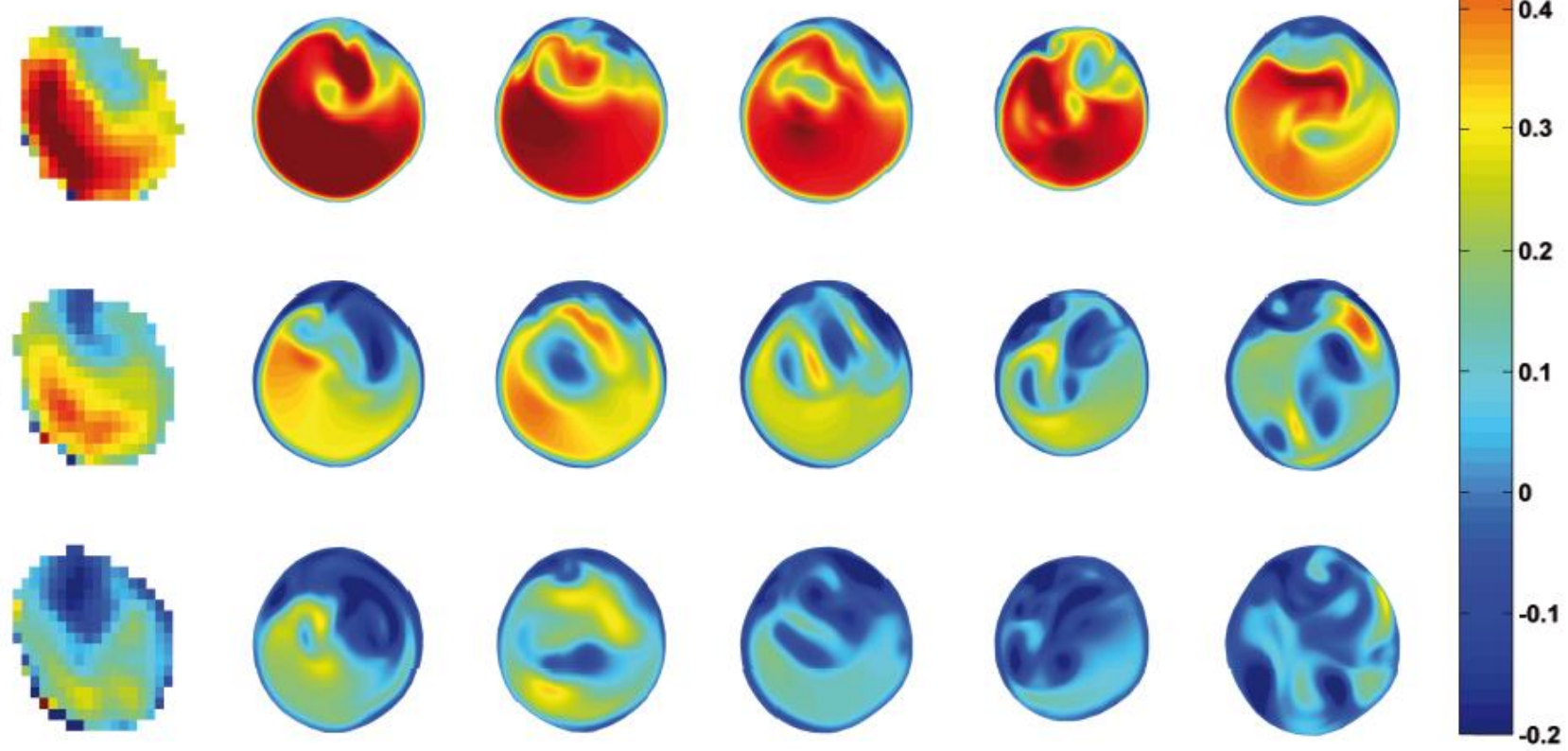

Time: $\mathbf{0 . 3 1}$ [s]
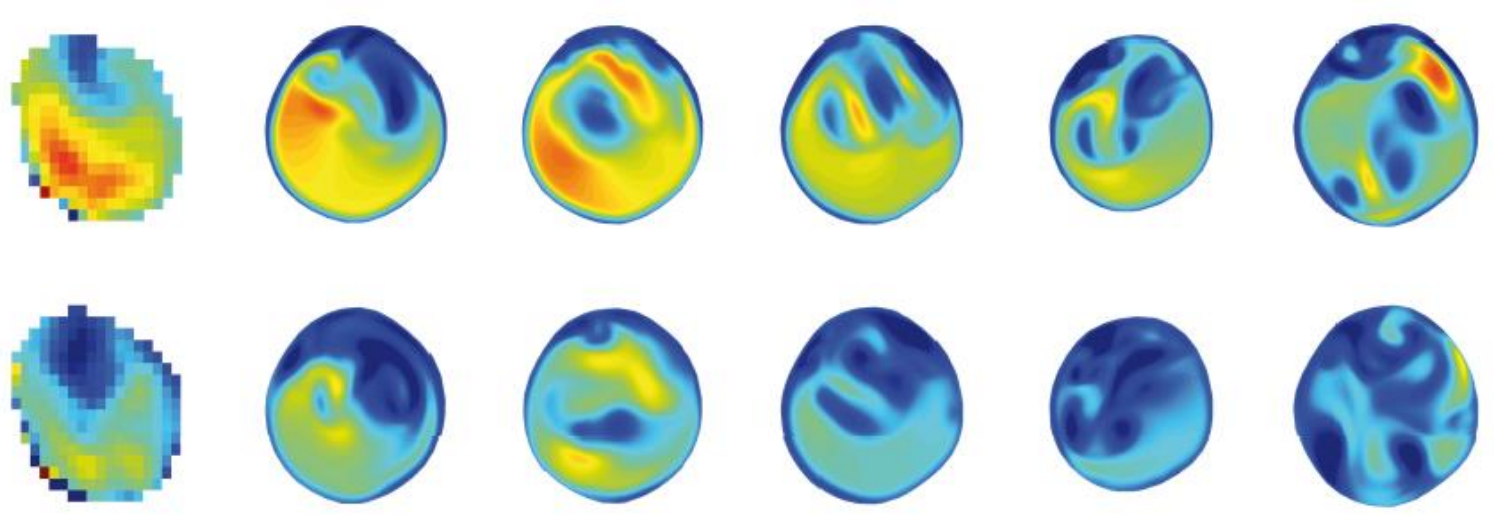

Time: $\mathbf{0 . 3 4}$ [s]
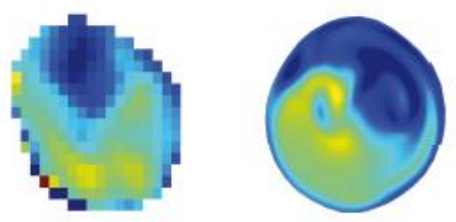
Figure6_Lantz.eps

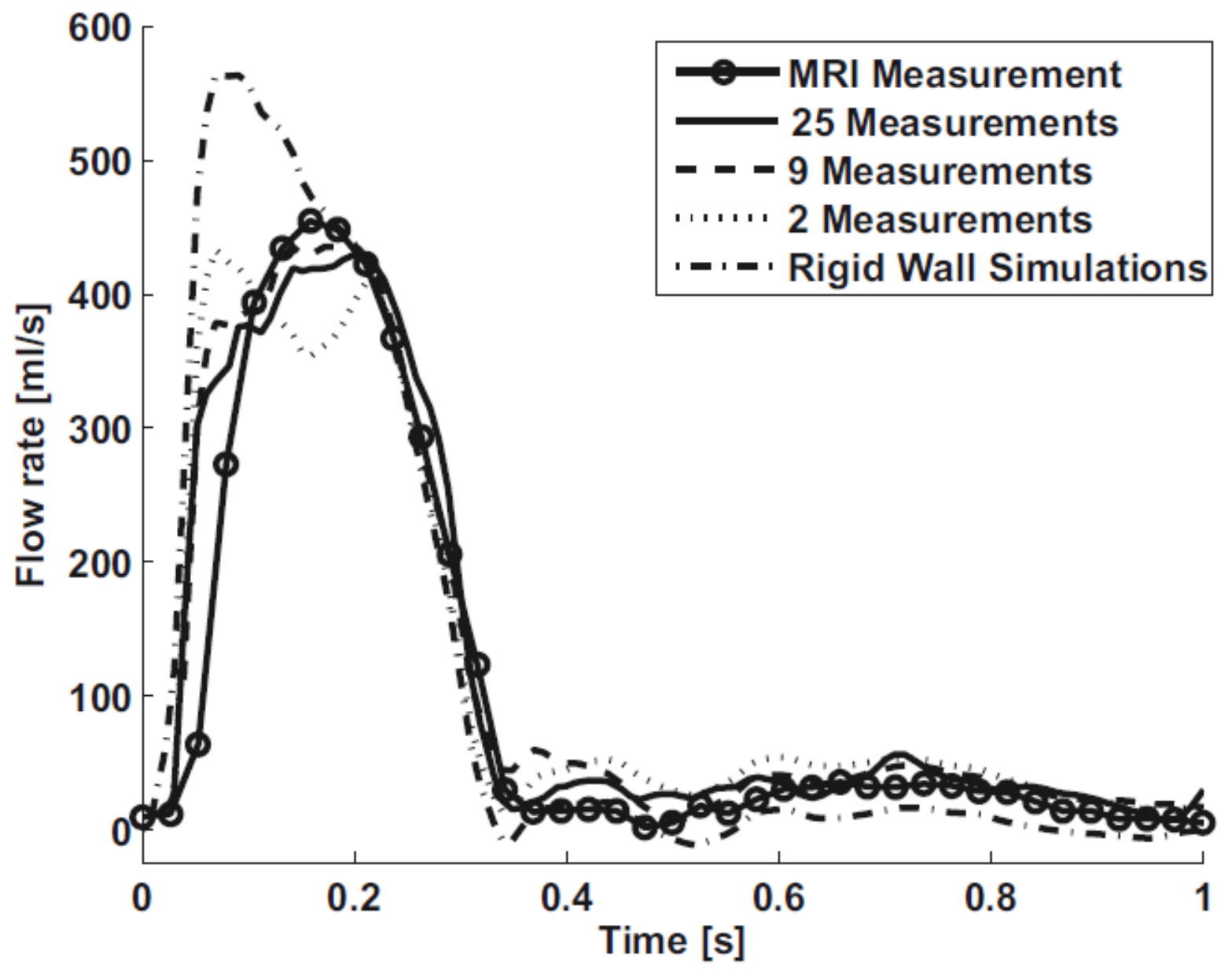


Figure7a_Lantz.eps
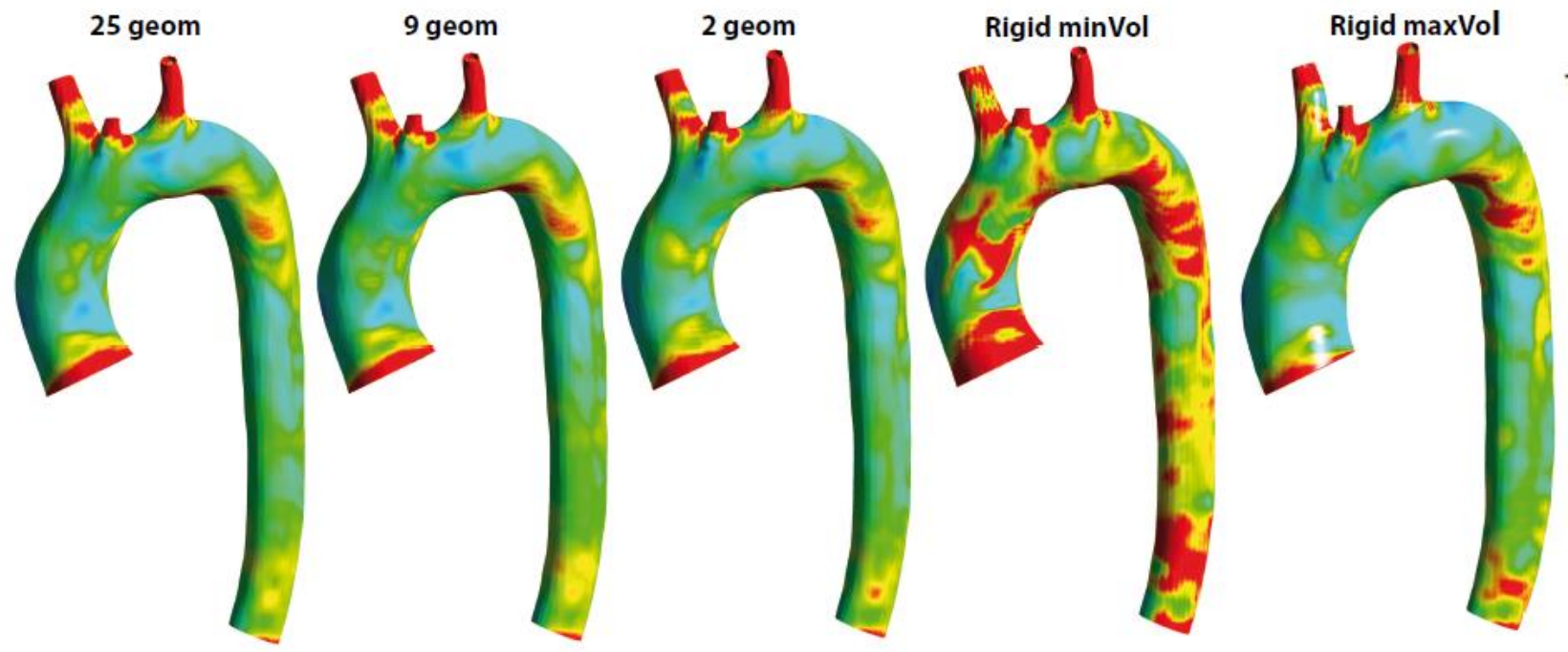

TAWSS [Pa]

$>5$

4

3

2

Figure7b_Lantz.eps
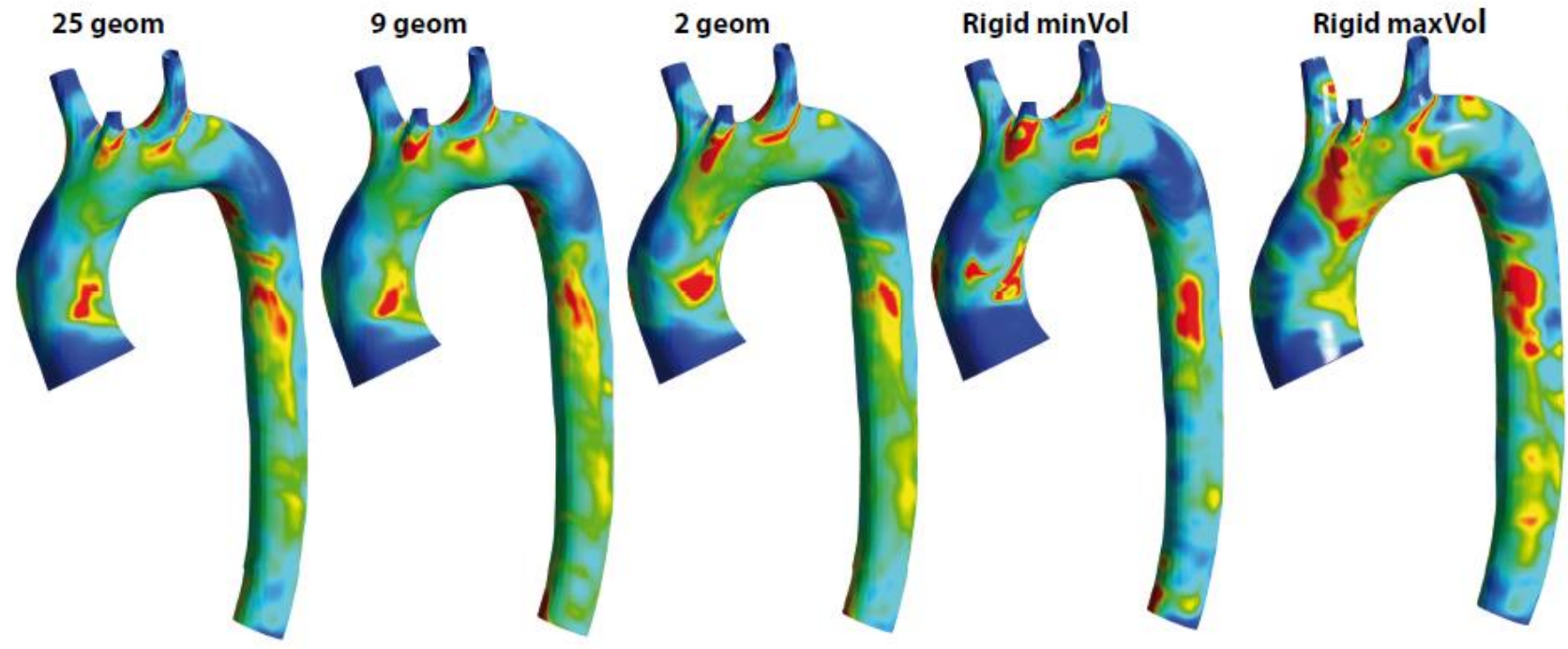

OSI [-]

$0.3-0.5$

0.2

0.1

0.0 
Figure8_Lantz.eps



\section{Figure captions}

Fig. 1: MRI-measured velocity profiles were prescribed as boundary conditions in the ascending aorta and in the branching vessels leaving the aortic arch; the resulting flow rates are here presented for each vessel

Fig. 2: Volume of the aorta over one cardiac cycle, where circles indicate each measured geometry for the base line case ( 25 measurements). The case with 9 measurements used every third geometry while the case with 2 measurements used the first geometry (minimum volume), and the 6th geometry (maximum volume). For all cases Piecewise Cubic Hermite Spline interpolation was used between the measurements

Fig. 3: Translation of the centroid in a cross-section in the ascending aorta. The cardiac cycle starts at $x, y=0$, and the centroid is then translated in a loop as a result of the beating heart and flow forces. The effect from using all 25 measurements compared to 9 or 2 measurements is apparent; most of the translation is lost if only 2 measurements are included in the simulation

Fig. 4: Comparison of velocity profiles from MRI measurements and CFD simulations in the descending aorta during systolic acceleration and peak flow rate. Body position is indicated in top left plot, with A: anterior, P: posterior, L: left and R: right.

Fig. 5: Comparison of velocity profiles from MRI measurements and CFD simulations in the descending aorta during systolic deceleration. Note the different color scale compared to Figure 4. Negative values indicate backflow, towards the heart. Same orientation as in Fig 4

Fig. 6: Comparison of the flow rate in a plane in the descending aorta for the different simulations. Notice how the rigid wall assumption affects the flow rate as there is no Windkessel effect in the model. For the cases with prescribed wall motion the flow rate agrees better with the MRI measurements

Fig. 7(a,b): Upper row, Left to right: Time-averaged wall shear stress for the cases with prescribed wall motion using 25 geometries, 9 geometries, 2 geometries, and rigid wall assumption at minimum volume and at maximum volume, 
respectively. Lower row, Left to right: Oscillatory shear index for the cases with prescribed wall motion using 25 geometries, 9 geometries, 2 geometries, and rigid wall assumption at minimum volume and at maximum volume, respectively

Fig. 8: Left to right: Wall shear stress magnitude at peak flow rate with prescribed wall motion using 25 geometries, 9 geometries, 2 geometries, and rigid wall assumption at minimum volume and at maximum volume, respectively 


\section{Supplementary material}

\section{Mesh interpolation method}

For interpolating mesh coordinates between two meshes, piecewise cubic hermite spline interpolation (PCHIP) was used. One advantage of using Cubic Hermite splines for interpolation over an arbitrary interval, is that is gives a smooth continuous function. Inputs are function values and the first derivatives at the interval limits. The MRI measurement had a time step of $0.044 \mathrm{~s}$, i.e. two subsequent meshes are $0.044 \mathrm{~s}$ apart, which is therefore the interpolation interval. With a time step of $0.0044 \mathrm{~s}$ in the CFD simulation, 9 intermediate coordinate locations between two meshes needed to be computed using the PCHIP method.

On an interval $\left[\mathrm{x}_{1}, \mathrm{x}_{2}\right]$ the PCHIP takes on the form:

$p(x)=h_{00}(t) p_{x 1}+h_{10}(t)\left(x_{2}-x_{1}\right) m_{x 1}+h_{01}(t) p_{x 2}+h_{11}\left(x_{2}-x_{1}\right) m_{x 2}$

where $\mathrm{p}(\mathrm{x})$ is the interpolated coordinate value, $\mathrm{t}=\left(\mathrm{x}-\mathrm{x}_{1}\right) /\left(\mathrm{x}_{2}-\mathrm{x}_{1}\right), \mathrm{x}$ is a location in $\left[\mathrm{x}_{1}, \mathrm{x}_{2}\right], \mathrm{p}_{\mathrm{x} 1}$ and $\mathrm{p}_{\mathrm{x} 2}$ are the coordinates at $x_{1}$ and $x_{2}, m_{x 1}$ and $m_{x 2}$ are the tangents at $x_{1}$ and $x_{2}$, and the $h$-terms are basis functions defined in the table below and shown in Figure A1. The interpolant will be a linear combination of the four basis functions.

Table A1: PCHIP basis functions

\begin{tabular}{|l|l|}
\hline$h_{00}(t)$ & $2 t^{3}-3 t^{2}+1$ \\
\hline$h_{10}(t)$ & $t^{3}-2 t^{2}+1$ \\
\hline$h_{01}(t)$ & $-2 t^{3}+3 t^{2}$ \\
\hline$h_{11}(t)$ & $t^{3}-t^{2}$ \\
\hline
\end{tabular}

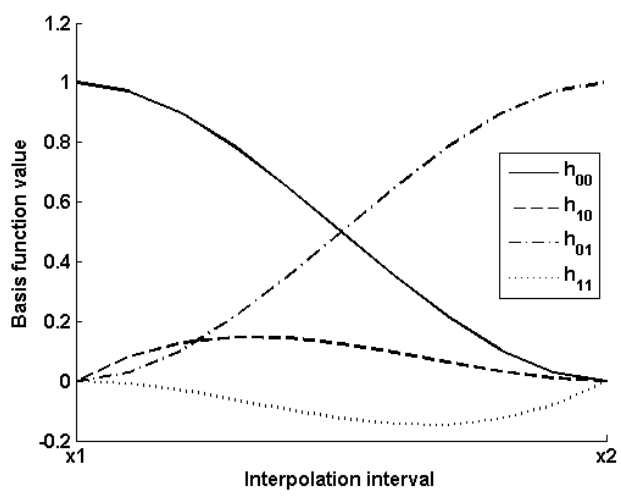

Figure A1: The four PCHIP basis functions over the interval $\left[\mathrm{x}_{1}, \mathrm{x}_{2}\right]$.

The tangents at $x_{1}$ and $x_{2}$ were obtained by central differencing, i.e. for interpolation between mesh \#2 and mesh \#3, $m_{x 1}$ was computed using mesh \#1 and \#3, while $m_{x 2}$ was computed using mesh \#2 and \#4.

When the interpolated coordinates were obtained, a built-in low-level subroutine in CFX (provided by ANSYS) moved the mesh from the original position to the interpolated position. This is only possible if the topology of the mesh remains the same for all meshes (i.e. same number of nodes and no "large" motion), as the node mapping must be consistent when moving the mesh. 